\title{
Discrimination of restrictions upon sequentially encoded information: Variable length periodicities*
}

\author{
IRWIN POLLACK $\dagger$ \\ University of Michigan, Ann Arbor, Michigan 48104
}

Two-state Markov sequences were encoded in the form of auditory pulse trains for forced-choice discrimination tests. Successive items were selected by block sampling from specified patterns, defined either in terms of a "string" with a beginning and an end or in terms of a "circle" without a beginning and end. The discriminability of such sequences is only partially related to constraints imposed upon the sampling procedures.

The detection of auditory periodicities is indeed a robust phenomenon. For example, Guttman and Julesz (1963) have shown that, with concentrated listening, one can detect the periodicity of repeated white-noise waveforms up to $4 \mathrm{sec}$ in duration. To specify digitally such a waveform with a bandwidth of $20 \mathrm{kHz}$ requires the enumeration of 160,000 numbers. Each of these numbers is effectively independent of all except its immediate neighbors, but there is a perfect correlation between Sample No. 1 and Sample No. 160,001; Sample No. 2 and Sample No. 160,002, etc. In informal tests with Dr. Lopes-Cardoza at the Instituut voor Perceptie Onderzoek, Eindhoven, Netherlands, I observed that the upper limit on periodicity detection is not changed appreciably by presentation of repeated noise samples against a range of low background noise levels. With interval-coded auditory pulse trains, 1 observed that partial periodicities, represented by the presentation of successive scramblings of a defined set of intervals, could also be detected (Pollack, 1968). And, most relevant to the present study, in informal tests at the Bell Telephone Laboratories, carried out with Miss S. Hanauer, partial periodicities could be observed by jittering the starting position of repeated noise patterns.

The present tests are concerned with the detection of partial periodicities, represented by block sampling from fixed patterns for interval-coded auditory pulse trains. The aim is to relate the observed discriminability among sequences with

\footnotetext{
* This research was supported by Grant GB 6148 from the National Science Foundation. The author is indebted to $\mathrm{K}$. Weidner, who supervised the experimental tests; to L. Radin, who wrote the PDP-8 computer program: and to R. Hsieh, who wrote the data processing program.

† Mailing address: Mental Health Research Institute, University of Michigan, Ann Arbor, Michigan 48104.
}

partial periodicities to the informational constraints imposed upon the sequences.

\section{GENERAL METHOD}

\section{Restriction of the Sequences}

The method for producing partial periodicities in the present study is outlined in Table 1. A "pattern" of Length $P$ is defined by a random scrambling of $\mathrm{P} / \mathrm{m}$ examples of each of $\mathrm{m}$ different states. For example, patterns of Length 8 can be obtained by scrambling 4 examples of each of 2 binary-coded states, by scrambling 2 examples of each of 4 binary-coded states, or by scrambling 1 example of each of 8 octal-coded states.

Perfect periodicity obtains when the pattern is repeated exactly: baaababb,baaababb, ... etc., or gchdbfae,gchdbfae, .... (The commas are to aid the reader; they were not represented in the sequences.) The conditions for perfect periodicity may be stated in terms of the following requirements: A pattern of $P$ units is regarded as a "string" with a defined beginning and termination; and that string is sampled in terms of a block of $P$ units.

A nonperfect, or partial, periodicity obtains when the pattern is not repeated exactly. The conditions for partial periodicity obtain when the pattern is regarded as a "circle" without a defined beginning and termination. In this case, we can sample from the pattern in blocks, B, of any size, including Length $P$ : gchdbfae,dbfaegch, ... etc. Or the conditions for partial periodicity obtain when we sample from the "string" pattern in blocks of less than P: hdbf,dbfa,chdb, .... Note that internal constraints-d followed by $b$-tend to be maintained with partial periodicities. Presumably, operations which preserve a greater proportion of internal constraints-larger block samples or sampling from string rather than from circular patterns-will yield more discriminable sequences.

The variables which defined the sequences were: $m$, the number of different states; $P$, the length of the pattern available for sampling; $S$, the smaller of two block-sampling lengths; $L$, the larger of two block-sampling lengths; and $N$, the total number of items in the sequence. The limits imposed by the generating program were: $2 \leqslant \mathrm{~m} \leqslant 8 ; \mathrm{P} \leqslant 300, \mathrm{P} / \mathrm{m}=$ integer; $\mathrm{N} \leqslant 625 ; \mathrm{N} / \mathrm{S}=$ integer $; \mathrm{N} / \mathrm{L}=$ integer.

Two series of tests, illustrated in Table 1, were run: The pattern was treated as a linear string (Variable Length Periodicity I, or VLPI), and the pattern was treated as a circle (Variable Length Periodicity II, or VLPII). The number of different starting positions is $(P-B+1)$ for VLPI and (P) for VLPII, where $B$ is the block-sampling length. The maximum number of different samplings from any pattern is $\mathrm{m}^{\mathrm{B}}$, or the number of starting positions, whichever is smaller. Other patterns may yield a smaller number of different samplings, e.g., only two different samples are yielded by $\mathrm{B}=2$ with the pattern 01010101

\section{Procedure}

Markov sequences were generated by a small digital computer (Digital Equipment Corporation PDP-8) in the form of electrical pulse trains. The pulse trains were converted to sound by earphones (Koss PRO-4) and were presented to listeners in forced-choice listening tests. The states were encoded in the form of the interpulse interval between successive pulses. For example, if State $A$ is represented by an interpulse interval of $0.4 \mathrm{msec}$ and State B is represented by an interpulse interval of $0.6 \mathrm{msec}$, the sequence of $\mathrm{ABA}$ is encoded as: pulse, wait $0.4 \mathrm{msec}$; pulse, wait $0.6 \mathrm{msec}$; pulse, wait $0.4 \mathrm{msec}$; pulse. In this example, the average interval, $\mathrm{IPI}_{c}$, is $0.5 \mathrm{msec}$, or 2,000 intervals per second; and the difference between interpulse intervals, DIPI, is $0.2 \mathrm{msec}$, or $40 \%$ of the mean interval.

An observation consisted of four pulse trains: three with the smaller sampling

Table 1

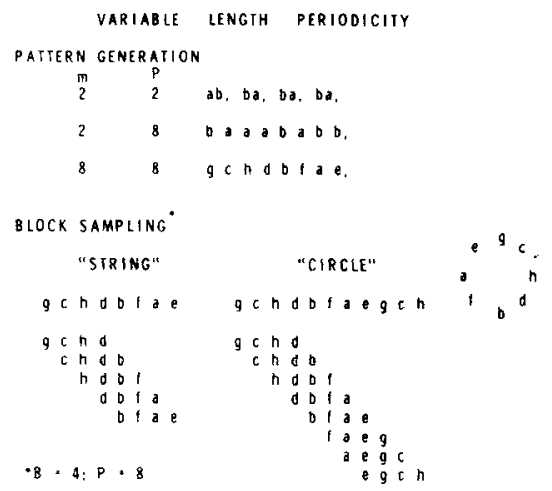




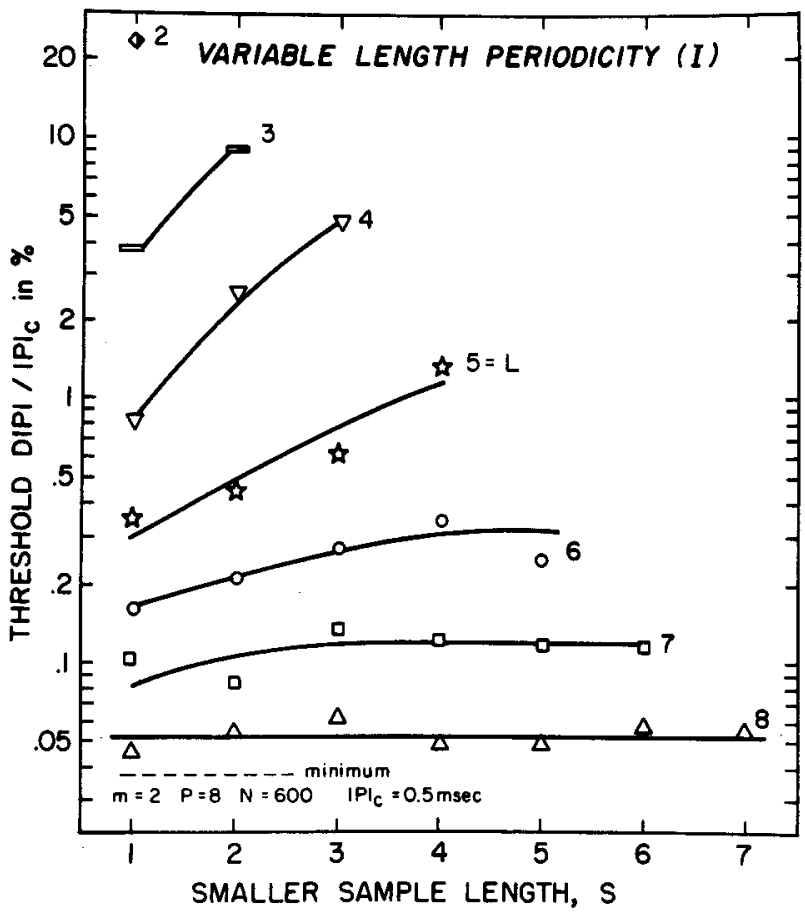

Fig. 1. Threshold difference in interpulse interval, DIPI, for binary patterns of Length 8 . The smaller sampling length is represented by the abscissa. The shading of the points in all figures reflects the proportion of terminated trials (see text). Method of sampling string patterns, VLPI.

length, $S$, and one with the larger sampling length, L. A single pattern, P, was generated for each observation. The listener heard four pulse trains. His task was to identify which one of the four pulse trains sounded different from the other three. When he responded correctly, an adaptive stimulus programming procedure of variable step size (Taylor \& Creelman, 1967) decreased DIPI-the difference in the interpulse interval between adjacent states. When he responded incorrectly, the adaptive procedure increased DIPI. The adaptive procedure converged upon $50 \%$ correct responses in the four-interval forced-choice (4IFC) test.

The use of DIPI as a dependent measure of discriminability is not obvious, except in the extreme case where DIPI $=0$. Here, all states are encoded identically, and all sequential constraints are nondiscriminable. We assume that the relative discriminability among the sequential constraints is related to the interval differences among the states. There is evidence to support this assumption with respect to the detection of scrambled periodicities in auditory pulse trains (Pollack, 1968).

When the number of different states is varied, we distinguish between DIPI or $\mathrm{DIPI}_{\mathrm{m}}$, the difference in interpulse interval between successive states, and $\operatorname{DIPI}_{R}$, the

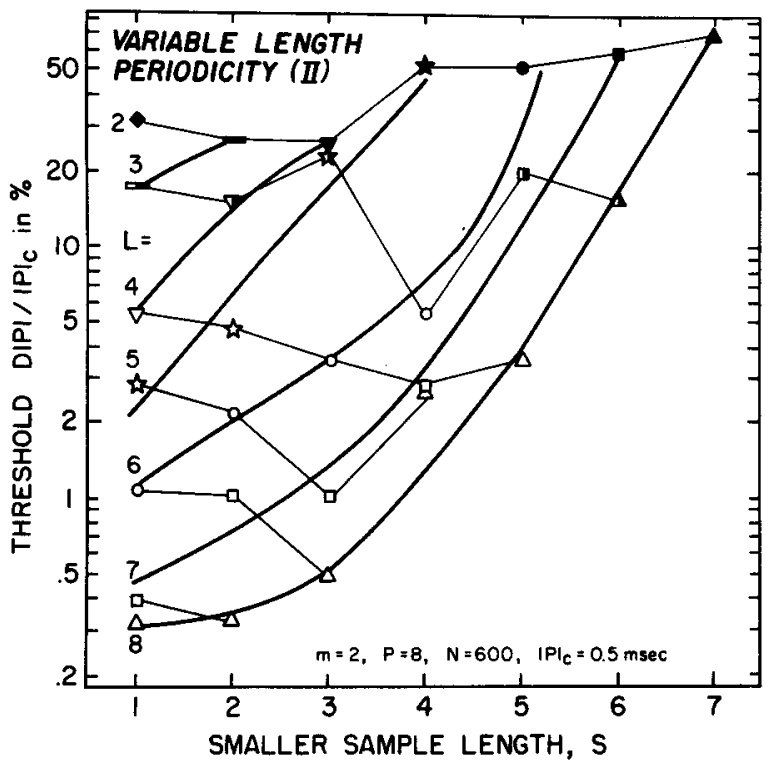

Fig. 2. As in Fig. 1, for method of sampling circle patterns, VLPII. The thin lines connect conditions of equal differences between longer ( $L$ ) and shorter (S) block-sampling lengths.

\section{RESULTS}

Figures 1 and 2 present the change in DIPI thresholds for the exhaustive combination of sample lengths for a pattern of eight binary-coded intervals. The abscissa is $\mathrm{S}$, the shorter sample length; the parameter is $L$, the longer sample length. The minimum threshold is one-half of the smallest unit of temporal control (0.375 microsec, or $3 / 16$ microsec in a period of 500 microsec, or $0.04 \%$ of the mean interval.

Figure 1 (VLPI) demonstrates an extremely wide-almost a three-decade -range of interstate thresholds as a function of both S and L. A strong interaction is evident. With strictly periodic signals $(L=P=8)$ and with nearly periodic signals $(L=7)$, there is little effect of the shorter sample length, S. Presumably, the periodic patterns, $\mathrm{L}=8$, and near-periodic patterns, $L=6,7$, are so distinctive that variations in $S$ are not effective in reducing thresholds. The shorter sample length, S, however, plays a substantially greater role under marginal conditions for discrimination, as when the larger sample length is not a substantial fraction of the entire pattern length, $L=3$ to 5 .

There is a substantial change in interstate thresholds in Fig. 2 for VLPII, relative to thresholds in Fig. 1 for VLPI. Since the underlying patterns were equivalent for specified conditions of $\mathrm{m}$ and $P$, the differences are presumably associated with the method of sampling the underlying patterns. In VLPI, the maximum number of different samples for Block Length $B$ is the smaller of 


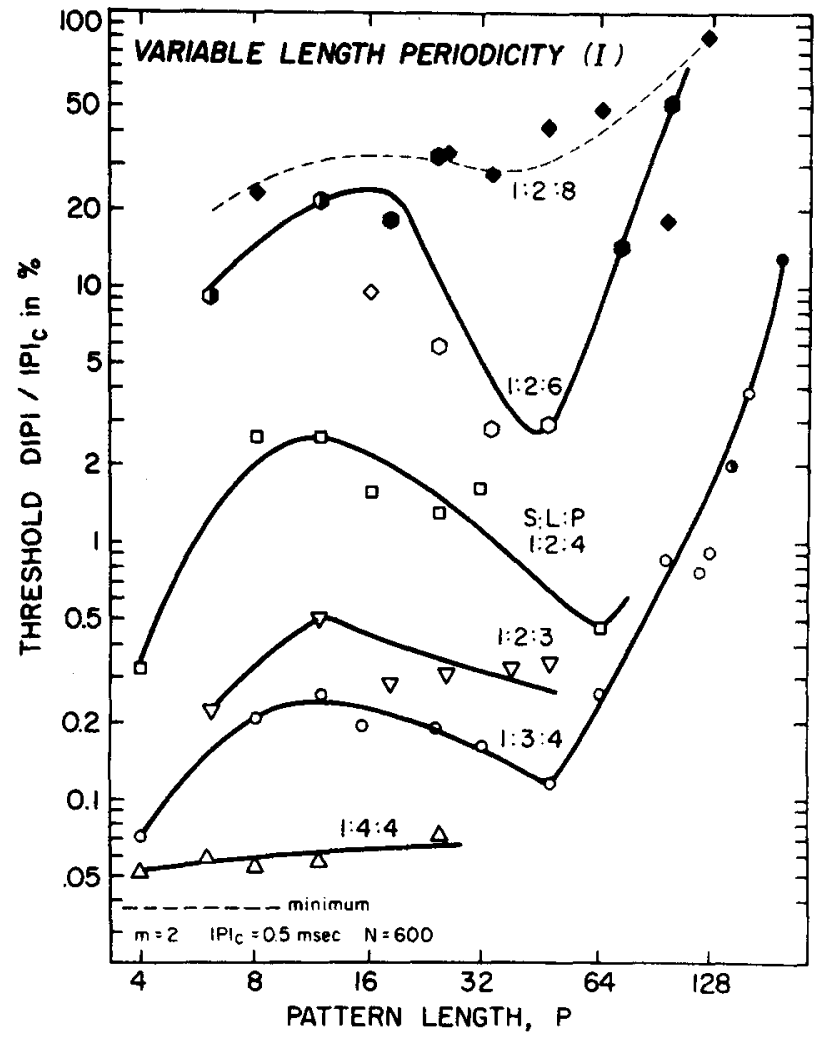

Fig. 3. Interstate difference thresholds for binary patterns of different length. The parameter is the ratio between the shorter block-sampling length, the longer block-sampling length, and the total pattern length. Method of sampling string patterns, VLPI.

$(P-B+1)$ and $\mathrm{m}^{B}$; in VLPII, the maximum number of different samples is the smaller of $P$ and $\mathrm{m}^{B}$. In Fig. 2, interstate thresholds are sensitive to $\mathrm{S}$ and $L$ over the entire range of both variables. The thin lines connect points of equal ( $L$ - S) differences. To a first approximation, a constant interstate discriminability threshold is associated with a given ( $L-S)$ difference, independently of the $\mathrm{L}$ and $\mathrm{S}$ sample lengths.

An exhaustive combination of conditions was also run for pattern lengths of 2, 4, and 6 units. The findings of Figs. 1 and 2 were also reflected in these tests.

The extension to longer underlying pattern lengths is examined in Figs. 3 (VLPI) and 4 (VLPII). The conditions are identified in terms of the $S: L: P$ ratio. For example, a ratio of $1: 3: 4$ with $P=12$ implies $S=3, L=9$, and $P=12$.

Consider first Fig. 3 (VLPI). A set of complex functions describes the results. Thresholds rise with increased pattern length (up to a pattern length of about 12 intervals), as might be expected on the basis of the increased number of possible selections per sampling and upon the decreased number of successive samplings within the total sequence. Then, not

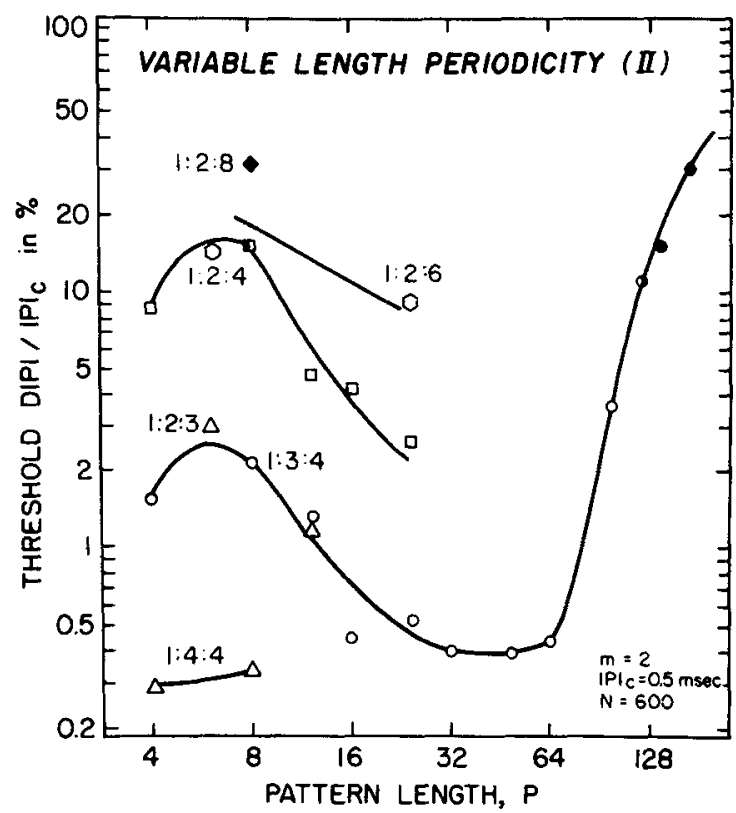

Fig. 4. As in Fig. 3, for method of sampling circle patterns, VLPII.

presumably because of an insufficient number of successive representations of the sampled pattern.

Figures 5 and 6 consider the role of the number of different states, $m$, of the sequences. Only data for a $S: L: P$ ratio of 1:3:4 are presented, although the same picture is maintained at other $S: L: P$ ratios. The insert of Fig. 5 reflects spacings among thresholds in accordance with two simple hypotheses: DIPI thresholds vary with the number of states to maintain a constant average deviation, A.D., about the mean; and DIPI thresholds maintain a constant range of interpulse intervals between the extreme states, DIPI $=(m-1)$ DIPI. At intermediate pattern lengths, DIPI thresholds appear to strike a compromise between these two hypotheses.

Figure 7 examines DIPI thresholds for fixed $S$ and $L$ sampling lengths as a function of the pattern length. The parameter is the sampling combination of $S$ and $\mathrm{L}$. Conditions with an $\mathrm{S} / \mathrm{L}$ ratio of 0.5 are examined in Fig. 7. The points connected by thin dashed lines in the upper left corner represent VLPII; the other points represent VLPI. For the latter condition, an interesting set of relationships is suggested by the successive curves. On the double-logarithmic plot, a series of near-power functions are obtained over almost the entire range of available thresholds. Minimum thresholds are obtained with VLPI when $\mathrm{L}=\mathrm{P}$; maximum thresholds are obtained when the S:L:P ratio is about $1: 2: 12$. Moreover, successive multiples of $\mathrm{S}$ and $\mathrm{L}$ produce nearly equivalent shifts in the functions: as from 1-2 to $2-4$ to $4-8$ to $8-16$. 

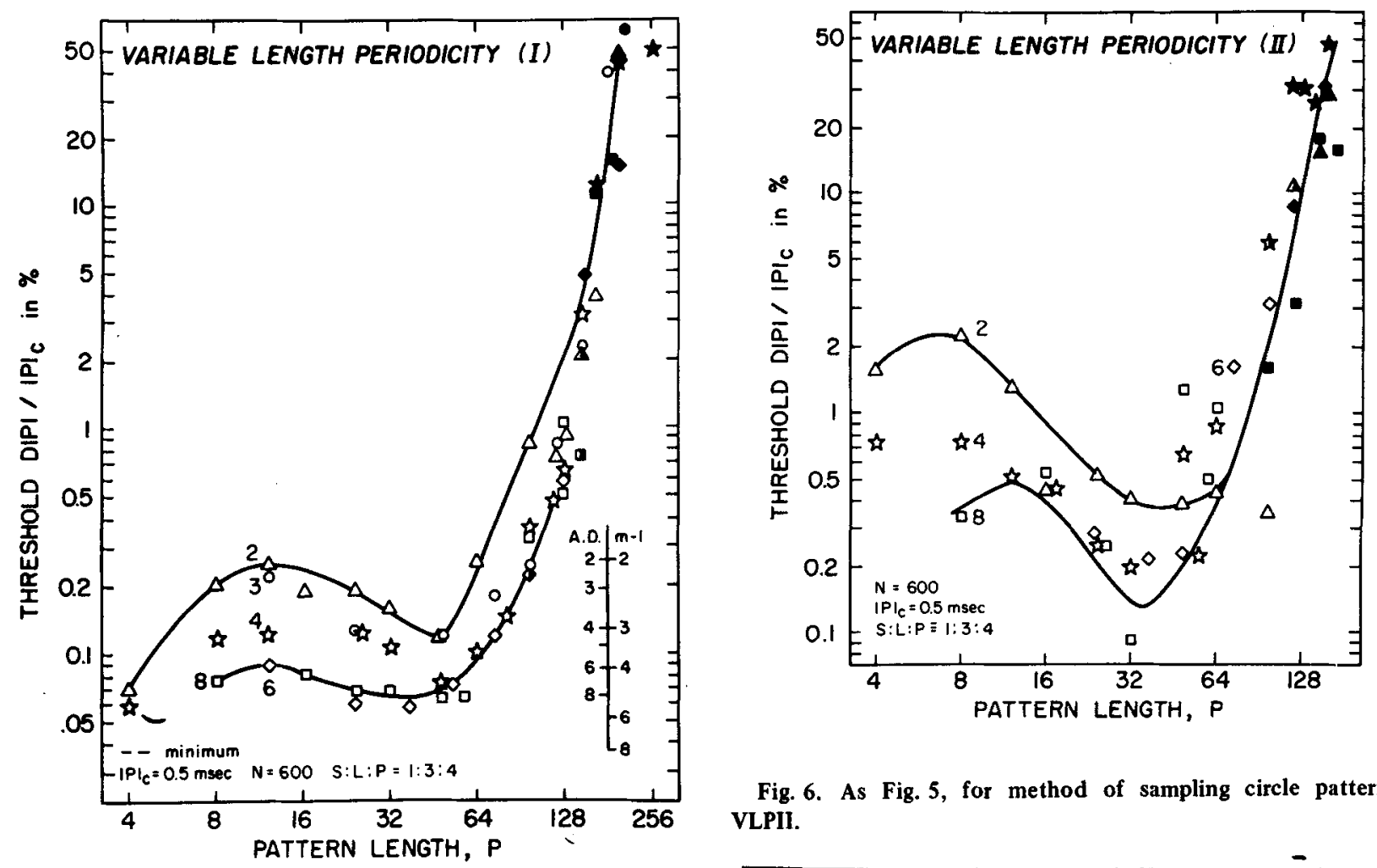

Fig. 6. As Fig. 5, for method of sampling circle patterns, VLPII.

Fig. 5. Interstate difference thresholds for multistate patterns of different length. The parameter is the number of different states in the sequence. The insert represents the expected spacing 50 among thresholds across different number of states under two hypotheses: The average deviation, A.D., among the various interpulse intervals is constant, or the range of interpulse intervals, DIPI $_{R}=(m-1) D I P I$, is constant. Method of sampling string patterns, VLPI.

An insufficient range of conditions was available from VLPII to test the effect of pattern length. From the few results (with thin dashed lines) available, thresholds appear to differ both qualitatively and quantitatively under VIPII at least for short patterns.

We previously noted a sharp rise in DIPI thresholds with longer pattern lengths (Figs. 3-6) or, alternatively, with a fixed S:L:P ratio with longer block-sampling lengths. The number of representations of each underlying pattern by a block sampling of Length $B$ within the total sequence of length, $N$, is N/B times. Thus, one can examine the effect of the number of representations of each pattem by varying either $\mathrm{N}$ or $\mathrm{B}$.

The previous tests employed sequences of 600-625 intervals. The tests of Figs. 8 and 9 examine the role of the length of the total sequence. For an $S: L: P$ ratio of $1: 3: 4$, the previously obtained complex functions are observed for a sequence length, $\mathrm{N}$, of 576 intervals. As the total sequence length is decreased, fewer and fewer samplings of the pattern are presented to the listener. The loss in

Fig. 7. Interstate difference thresholds for binary interval patterns with a constant $S / L$ ratio of $1: 2$. The abscissa is the length of the string pattern available for sampling. The parameter is the specific combination of $S$ and $L$. The thin dashed lines in the upper left represent the method of sampling circle patterns.

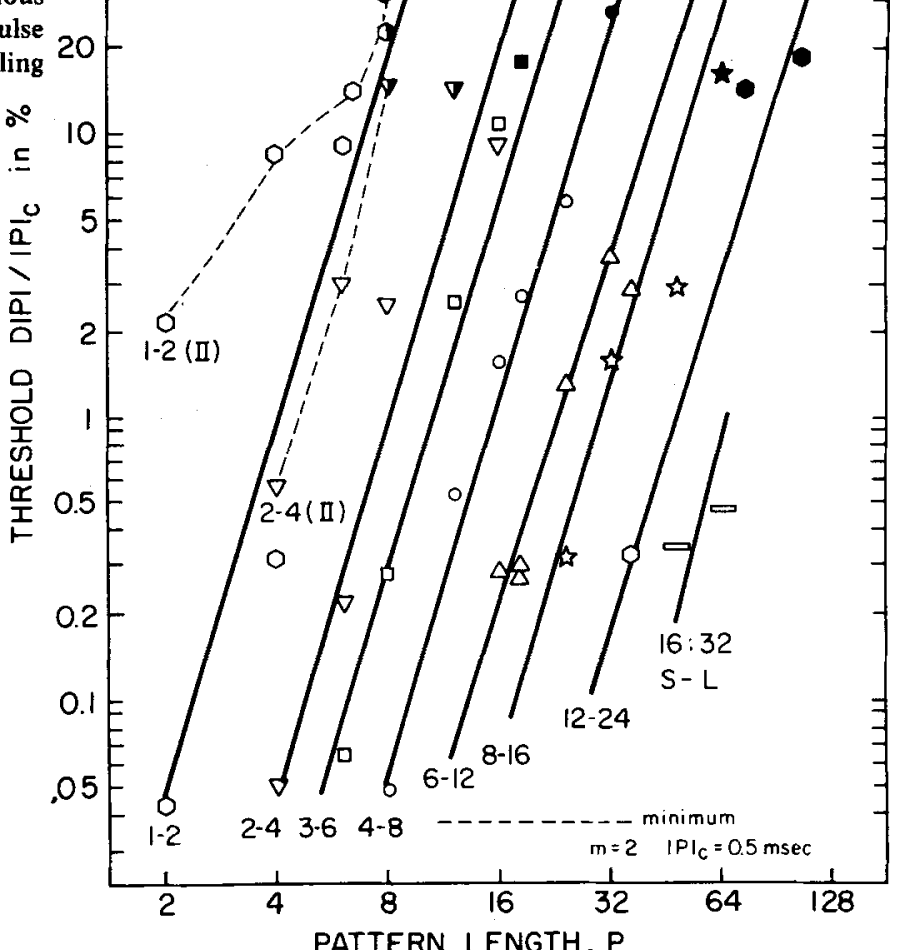




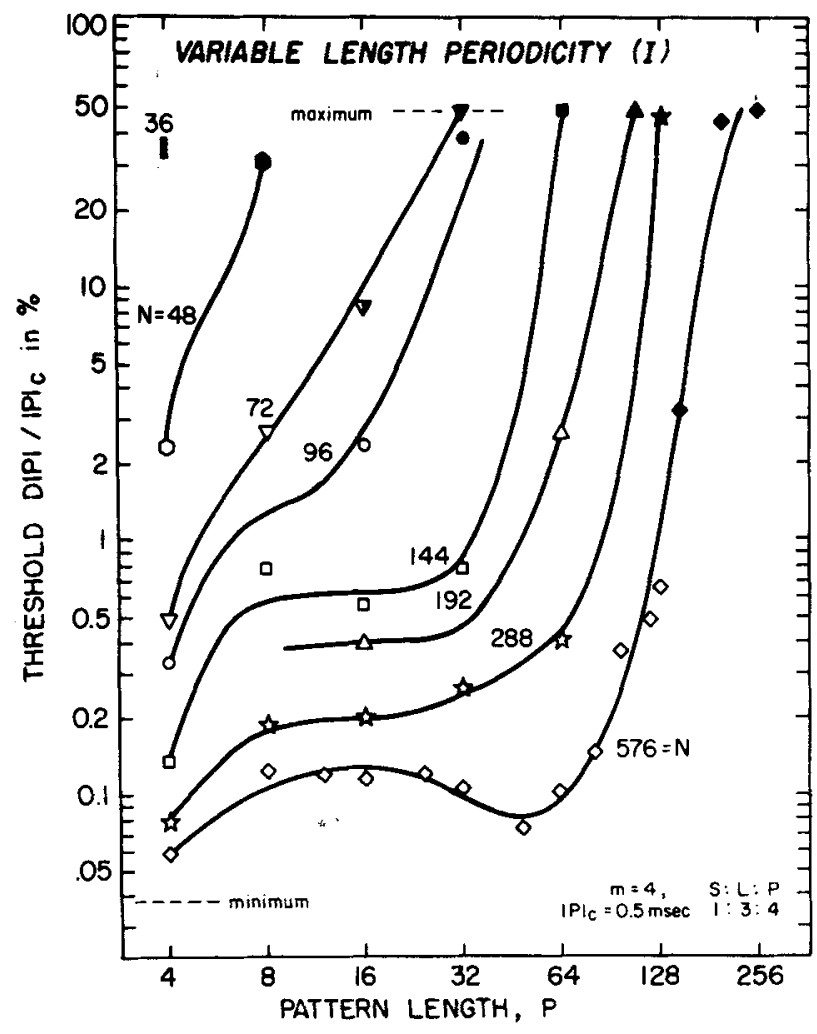

Fig. 8. Interstate difference thresholds for sequences of different length. The parameter is the total number of intervals in the presented pulse train, N. Method of sampling string patterns, VLPI.

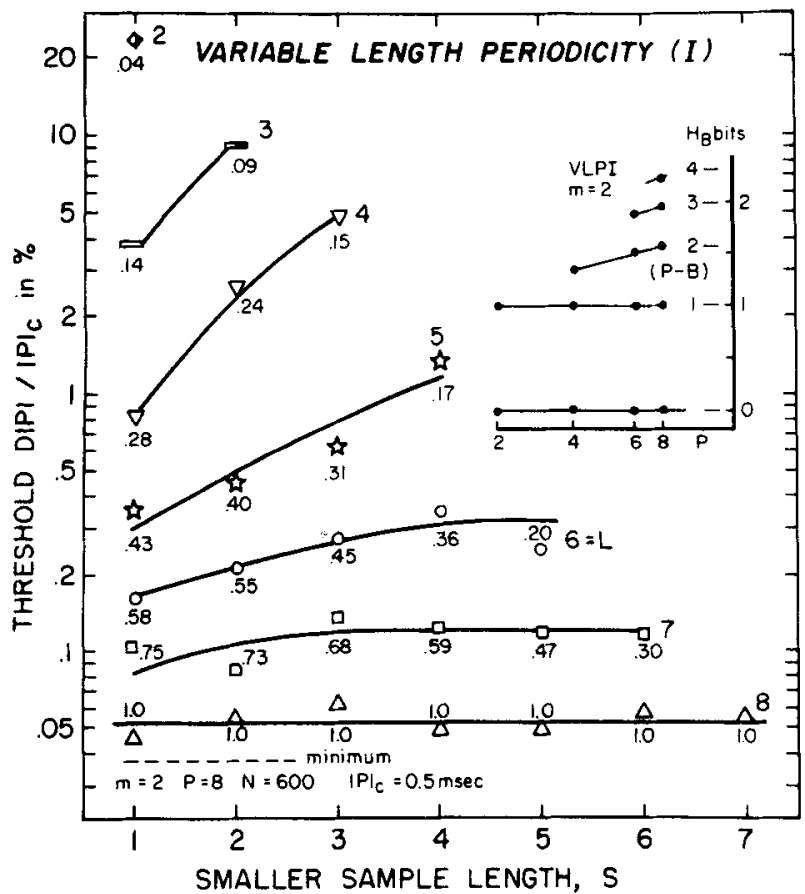

Fig. 10. Reexamination of Fig. 1 in terms of the unit informational difference ratio, calculated for the individual points. Method of sampling string patterns, VLPI.

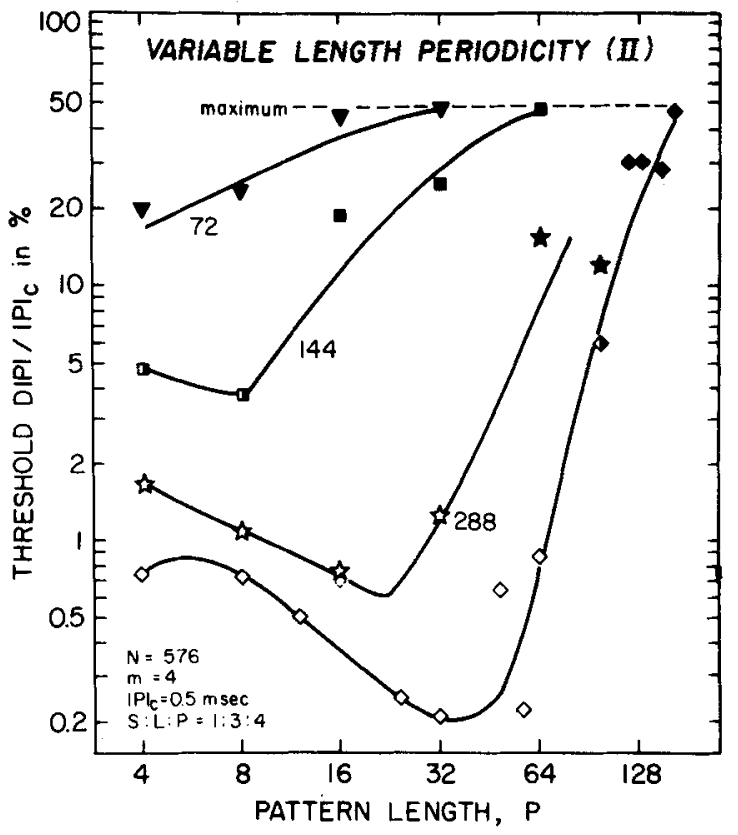

Fig. 9. As Fig. 8, for method of sampling circle patterns, VLPII.

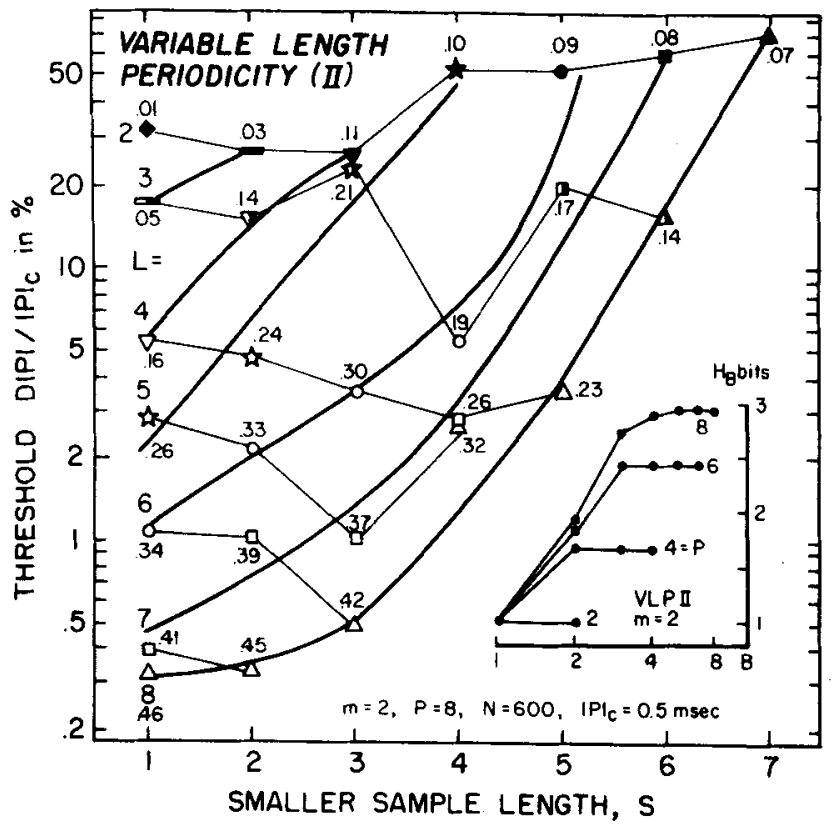

Fig. 11. As Fig. 10, for reexamination of Fig. 2. Method of sampling circle patterns, VLPII.

sensitivity is not a simple translation of the functions. Rather, the discriminability threshold function becomes more and more monotonic with pattern length for VLPI (Fig. 8) and with more rapid changes in sensitivity occurring with VLPII (Fig. 9).

DISCUSSION

The underlying hypothesis in the present 
tests (and in other tests in a related program of research, e.g., Pollack, 1968) is that discriminability thresholds for restricted sequences reflect the difference in informational uncertainty between the sequences. To a rough approximation, the underlying hypothesis is reflected in the data. Lowest thresholds obtain when $S=1$, with a random sampling among the $m$ states, is paired against $L=P$, with a strictly periodic sequence under VLPI. The former condition reflects the maximum uncertainty of specification of the sequences; the latter condition reflects the minimal uncertainty of specification. Deviation from the extremes-either by a decrease in uncertainty with a larger $S$ sampling or, more effectively, by an increase in uncertainty with a smaller L sampling from a fixed pattern length, P-results in higher thresholds. In either case, the difference in uncertainty decreases. Moreover, discrimination thresholds under VLPI are substantially lower than under VLPII, often by several decade units, e.g., 7-8-8 in Fig. 1 vs $7-8-8$ in Fig. 2. The greater uncertainty in specifying the patterns in VLPII is presumably also reflected in the larger thresholds.

For patterns of a given length, a modified form of the informational measure appears to account for a healthy portion of the total variance. Figures 10 and 11 reexamine the results of Figs. 1 and 2 in terms of the ratio: $\left(\mathrm{H}_{\mathrm{S}}^{\prime}-\mathrm{H}_{\mathrm{L}}^{\prime}\right) /\left(\mathrm{H}_{\mathrm{S}}^{\prime}+\mathrm{H}_{\mathrm{L}}^{\prime}\right)$, where $\mathrm{H}_{\mathrm{S}}^{\prime}$ and $\mathrm{H}_{\mathrm{L}}^{\prime}$ represent the average informational uncertainty, per unit of the sampled sequence, associated with the smaller and with the larger block-sampling lengths, respectively. This measure is essentially the ratio of the unit difference in uncertainty relative to (twice) the unit average uncertainty. The measure associated with each $S, L$ pair of Figs. 1 and 2 is presented in Figs. 10 and 11. The measures were derived from block sampling from all possible binary patterns of Lengths $2,4,6$, and 8 , with the restriction that each of the binary states occur equally often. The results of the samplings are shown in the upper-right inserts of Figs. 10 and 11. For example, with VLPI (Fig. 10), there is 0 bits of uncertainty with $(P-B=0)$, since the sequence is completely determined after specification of the initializing sequence. And there are two equally likely selections, or 1 bit of uncertainty, with $(P-B=1)$. Except for sequences with internal periodicities, e.g., $01010101 \ldots$, the overall uncertainty quickly approaches the limit of $\log _{2}(P-B+1)$, where $(\mathrm{P}-\mathrm{B}+1)$ is the number of starting positions under VLPI. Similarly, with VLPII (Fig. 11), the functions quickly approach a limit related to $P$, specifically $\left[(\mathbf{P}-1) \log _{2} \mathbf{P}+1\right] / \mathbf{P}$. The informational unit uncertainty is obtained by dividing the block uncertainty, $\mathrm{H}_{\mathrm{B}}$, by the block length, $B$, where $B$ is equal to $S$ or $L$.

The unit ratio uncertainty measure is nearly inversely monotonic with the DIPI thresholds within VLPI (Fig. 10) and within VLPII (Fig. 11). Moreover, nearly equivalent DIPI thresholds are associated with a fixed level of the measure across VLPI and VLPII.

The detailed quantitative relation between sequential uncertainty and discrimination thresholds, however, has not been further pursued because of the complex shape of the discrimination functions with pattern length (e.g., Figs. 3 and 4). The shape of these functions is not reflected in an informational analysis of the sequence generators, although the large differences between extremely short and extremely long pattern lengths are adequately reflected. It is noted that the unit informational ratio is relatively constant for a fixed S:L:P ratio. For example, over the intermediate range of pattern lengths where the discriminability function is decreasing-approximately between 16 and 48 units for VLPI-the unit information ratio varies from about 0.65 to 0.62 for an $S: L: P$ ratio of $1: 3: 4$.

We are thus left in the position of entertaining a reasonable hypothesis which reflects many of the gross main effects in the present tests, as well as in predicting the effects of the other forms of sequential restrictions, but which does not reflect important details of imperfect periodicities-such as the improvement in discriminability for intermediate pattern lengths. Specifically, the auditory system appears to make an incomplete trade-off between the length of the sampling of the underlying pattern and the number of successive samplings of the underlying pattern. The overall information measure makes a complete trade-off between these variables, and therein may lie the seed of its inadequacy.

\section{REFERENCES}

GUTTMAN, N., \& JULESZ, B. Lower limits of auditory periodicity analysis. Journal of the Acoustical Society of America, 1963, 35, 610(L).

POLLACK, I. Periodicity discrimination for auditory pulse trains. Joumal of the Acoustical Society of America, 1968, 43, 1113-1119.

TAYLOR, M., \& CREELMAN, D. PEST: Efficient estimates on probability functions. Journal of the Acoustical Society of America, 1967, 41, 782-787.

(Accepted for publication July 29, 1970,) 